\title{
Atomically Layered Helium Films at Ultralow Temperatures: Model Systems for Realizing Quantum Materials
}

\author{
John Saunders ${ }^{1} \cdot$ Brian Cowan $^{1} \cdot$ Jan Nyéki ${ }^{1}$ \\ Received: 28 December 2019 / Accepted: 13 March 2020 / Published online: 13 April 2020 \\ (c) The Author(s) 2020
}

\begin{abstract}
This year is also the 50th anniversary of the discovery of exfoliated graphite as a particularly uniform substrate (Thomy and Duval in J Chim Phys 66:1966, 1969. https://doi.org/10.1051/jcp/196966s21966, J Chim Phys 67:286, 1970. https://doi. org/10.1051/jcp/1970670286, J Chim Phys 67:1101, 1970. https://doi.org/10.1051/ jcp/1970671101). In this article, we focus on the study of helium films on graphite-based substrates at ultralow temperatures. We provide a flavour of the historical development of this subject and a perspective on the current status. We discuss how atomically layered helium films provide model systems for the realization of a broad range of quantum materials of generic significance. Future prospects arising from new techniques and new substrates will also be discussed.
\end{abstract}

Keywords Two dimensions - Strongly correlated fermions · Frustrated magnetism • Quantum spin liquid · Heavy fermion · Quantum criticality · Intertwined order · Supersolid · Topological superfluidity

\section{Introduction}

Helium films adsorbed on graphite substrates provide an extraordinary range of different systems with which to address questions of central importance in the field of quantum materials [1-3]. The flexibility derives from the ability to create a range of composite substrates by preplating the graphite surface. We can study both ${ }^{3} \mathrm{He}$ and ${ }^{4} \mathrm{He}$, and ${ }^{3} \mathrm{He}$ on a superfluid ${ }^{4} \mathrm{He}$ film. The films are readily cooled into the microkelvin temperature régime, revealing new emergent quantum states. We provide a perspective-style overview of progress and future prospects on: 2D Fermi systems; coupled 2D fermion-boson systems; Mott-Hubbard transition in 2D; heavy fermion quantum criticality; frustrated magnetism and quantum spin liquid; 2D supersolid.

John Saunders

J.Saunders@rhul.ac.uk

1 Department of Physics, Royal Holloway University of London, Egham, Surrey TW20 0EX, UK 


\section{Some History}

The key trigger to advance the study of helium physisorbed on graphite was the commercial availability of exfoliated graphite (Grafoil) as a high-quality substrate, with nearly ideal and clean surfaces, not subject to contamination [4]. The high specific surface area of $20 \mathrm{~m}^{2} / \mathrm{g}$, arising from atomically flat basal planes exposed by chemical exfoliation of natural graphite crystals, permitted heat capacity and vapour pressure studies. The primary focus of the first work on helium was to address: the existence or otherwise of crystalline order in twodimensional solids; phase transitions in commensurate phases stabilized by the honeycomb structure of substrate carbon atoms [5]. Other adsorbates such as Ne, $\mathrm{Xe}, \mathrm{Kr}, \mathrm{H}_{2}, \mathrm{D}_{2}, \mathrm{~N}_{2}, \mathrm{CO}$ have been extensively studied [6]. Synchrotron X-ray scattering and neutron scattering have been used to determine structure and to study 2D melting transitions [7].

The highly quantum nature of helium due to its large zero-point motion, consequent on its small mass, and weak interatomic interactions, gives rise to many unique features. The minimum temperature accessible in the earliest studies of helium on graphite was around $0.3 \mathrm{~K}$, achieved by pumping on ${ }^{3} \mathrm{He}$. This was sufficient to establish many features of the sub-monolayer phase diagram by heat capacity, vapour pressure and pulsed NMR. At the lowest coverage, a fluid phase was seen; studies of interactions in the fluid phase were restricted to an analysis in terms of virial expansions. A striking feature was the formation of a $\sqrt{3} \times \sqrt{3}$ commensurate solid, registered with the substrate, in which $1 / 3$ of the graphite basal plane hexagons are occupied. This was identified from a sharp melting peak near $3 \mathrm{~K}$; the width of this anomaly is reduced when using a substrate of larger platelet size (ZYX exfoliated graphite) $[8,9]$.

Studies by pulsed NMR were important to access the correlation time of the atomic quantum motion, present even in the $2 \mathrm{D}$ solid, and to distinguish between phases. Measurements of the spin-lattice relaxation time and intrinsic spin-spin relaxation time $T_{2}$ determine the spectral density of local field fluctuations. This clearly identified a transition from fluid to incommensurate solid (at $1 \mathrm{~K}$ ) on increasing coverage [10]. In the $2 \mathrm{D}$ incommensurate solid, $T_{2}$ decreases dramatically with increasing density, reflecting the exponential reduction in exchange coefficient and hence weakening of motional narrowing of the NMR line. It exhibits a sharp cusp-like minimum when it becomes energetically favourable for atoms to enter a second layer, where they are highly mobile. The correlation time observed in the incommensurate solid reflected a single effective ${ }^{3} \mathrm{He}-{ }^{3} \mathrm{He}$ exchange rate [11]. At lower temperatures and densities between $\sqrt{3} \times \sqrt{3}$ commensurate solid and incommensurate solid, a further phase was seen in heat capacity measurements on both ${ }^{3} \mathrm{He}$ and ${ }^{4} \mathrm{He}$ [12], subsequently identified as a domain wall solid [13, 14].

In the 1970s, improvements in dilution refrigerator technology led to the development of platforms for cooling quantum materials to low $\mathrm{mK}$ temperatures. The mid-1980s saw the remarkable demonstration that ${ }^{3} \mathrm{He}$ on exfoliated graphite could be cooled to these temperatures [15]. Technically, this depends on the 
semimetal nature of graphite, and the ability to diffusion bond Grafoil to silver foils, which provided a high thermal conductivity thermal link to the ultralowtemperature platform. The first result used continuous wave NMR to demonstrate the evolution of a ${ }^{3} \mathrm{He}$ film from a sub-monolayer paramagnetic solid; growth of second layer fluid which solidified just before promotion to a third layer; emergence of a strong peak in ferromagnetic exchange around 2.5 layers $[15,16]$. This opened the door to the investigation of ${ }^{3} \mathrm{He}$ by thermodynamic measurements in both degenerate 2D Fermi liquids, to study strongly correlated fermions, and in 2D solid phases to study the consequences of frustrated exchange interactions in an ideal 2D nuclear magnetic system.

Around the same time, the technology of nuclear adiabatic demagnetization was refined to cool ${ }^{3} \mathrm{He}$ into the superfluid phases. NMR studies of samples in which the superfluid ${ }^{3} \mathrm{He}$ was imbibed into stacks of mylar sheets (to control superfluid texture) showed up a surface contribution to the magnetism that arose from a surface boundary layer of solid ${ }^{3} \mathrm{He}$ [17]. This made a large contribution to the magnetic susceptibility at low temperatures, close to Curie law but with evidence for weak ferromagnetic exchange. This subject developed into extensive studies of the surface magnetism of ${ }^{3} \mathrm{He}$ within exfoliated graphite [18] extended to low magnetic field by the use of SQUID NMR [19] and as a function of liquid pressure which tunes the number of solid layers [20]. Nuclear adiabatic demagnetization platforms have subsequently been used extensively to study helium films on graphite. This régime is the main focus of the present article.

An alternative approach to the study of thin helium films has been physisorption on heterogeneous substrates such as mylar and nuclepore filter paper. In this case, the non-uniformity of the surface binding potential leads to localized helium at low coverages forming a so-called dead layer. At higher coverages, ${ }^{4} \mathrm{He}$ is mobile and covers the entire surface. This is in stark contrast to liquid ${ }^{4} \mathrm{He}$ films on graphite, which exhibits a gas-liquid transition in the second layer and above, with 2D liquid puddles at the self-bound density of order $4 \mathrm{~nm}^{-2}$. [In the first ${ }^{4} \mathrm{He}$ layer, the coexistence is between gas and $\sqrt{3} \times \sqrt{3}$ commensurate solid]. As gas-liquid condensation is inhibited on mylar, it was possible to study the onset of superfluidity in atomically thin ${ }^{4} \mathrm{He}$ films and observe the predicted Berezinskii-Kosterlitz-Thouless (BKT) transition [21-23], where the destruction of superfluidity with increasing temperature arises from the unbinding of vortex-antivortex pairs. These experiments rely on precise torsional oscillator techniques to measure the superfluid response. For the application of this method to the study of ${ }^{4} \mathrm{He}$ on graphite, see [24-26].

\section{Atomically Layered Helium Films at Ultralow Temperatures}

In this section, we briefly outline the landscape of atomically layered helium films on graphite, with an emphasis on the role of preplating to create composite substrates. Following this section, the article is organized by the class of quantum material under investigation, making use of these various preplatings. 


\subsection{Monolayer ${ }^{3} \mathrm{He}$ Films}

At low coverages, ${ }^{3} \mathrm{He}$ atoms (up to of order $5 \%$ of monolayer coverage) are localized by residual substrate heterogeneity [27]. More recent heat capacity measurements down to $2 \mathrm{mK}$ show that the ${ }^{3} \mathrm{He}$ monolayer condenses into a self-bound $2 \mathrm{D}$ liquid puddle with density around $0.8 \mathrm{~nm}^{-2}$ [28]. A similar gas-liquid condensation is also seen in the second and third layer [28]. Above a density of around $8 \mathrm{~nm}^{-2}$ the first layer forms a solid on a triangular lattice incommensurate with the graphite substrate. At completion, this is a relatively compressed 2D solid, with weak exchange interactions, essentially paramagnetic. The $\sqrt{3} \times \sqrt{3}$ registered solid forms at $6.3 \mathrm{~nm}^{-2}$. Between this and $8.0 \mathrm{~nm}^{-2}$ new registered structures and possible domain wall solids are expected $[13,14]$. Studies reveal a peak in ferromagnetic exchange at a coverage of $7.5 \mathrm{~nm}^{-2}[29,30]$. Furthermore, in this coverage régime the heat capacity shows an anomalous power-law temperature dependence from $100 \mu \mathrm{K}$ to $10 \mathrm{mK}[31,32]$. These intriguing observations are not fully accounted for and we believe the nuclear magnetism in this régime is worthy of further exploration to achieve a better understanding of the interplay of atomic exchange with putative domain wall-like structures.

\subsection{Multilayer ${ }^{3} \mathrm{He}$ Films}

Helium films exhibit multilayer growth on the atomically flat surface of graphite. This is graphically demonstrated experimentally by low-temperature vapour pressure isotherms, which show steps in chemical potential as a function of coverage [33] and theoretically by first principles calculations [34, 35]. Studies of multilayer ${ }^{3} \mathrm{He}$ films have been reviewed in $[16,36]$.

\subsection{Preplating}

In the study of ${ }^{3} \mathrm{He}$ films, the graphite surface can be preplated with a number of ${ }^{4} \mathrm{He}$ atomic layers, the choice of which leads to a different composite substrate. This preplating relies on the higher binding energy of the ${ }^{4} \mathrm{He}$ atom to the graphite surface by the helium-graphite attractive potential, due to its higher mass and hence lower zero-point energy. For example, the magnetic properties of the second layer of ${ }^{3} \mathrm{He}$ are best studied by replacing the completed first solid ${ }^{3} \mathrm{He}$ layer, which is paramagnetic, by non-magnetic solid ${ }^{4} \mathrm{He}$ [37]. This forms with a slightly higher density than the first ${ }^{3} \mathrm{He}$ layer. The perturbation on "second layer" properties is expected to be weak since exchange between the first and second ${ }^{3} \mathrm{He}$ layers is small, of the order of the dipolar interaction, as revealed by a two component NMR lineshape when both layers are solid.

If the graphite is preplated with a bilayer of solid ${ }^{4} \mathrm{He}$, the first ${ }^{3} \mathrm{He}$ layer only solidifies under the influence of the second ${ }^{3} \mathrm{He}$ layer; this leads to heavy fermion physics and Kondo breakdown quantum criticality. Beyond bilayer solid ${ }^{4} \mathrm{He}$, 
subsequent preplating ${ }^{4} \mathrm{He}$ layers are superfluid. This provides a flexible substrate to study $2 \mathrm{D}$ fluid ${ }^{3} \mathrm{He}$, in which, however, fermion-boson coupling must be carefully taken into consideration.

Preplating with a solid HD bilayer gives another composite substrate $\left(\mathrm{H}_{2}\right.$ is avoided because of attendant ortho-para conversion and associated heat release). In this case, the first ${ }^{3} \mathrm{He}$ layer shows a density-driven Mott transition, into 2D quantum solid with stronger exchange interactions than observed in the "second layer" solid [38]. This arises because of lower ${ }^{3} \mathrm{He}$ solid density, attributed to commensuration with the HD bilayer solid on graphite. While the use of inert gases (Ar, Ne, $\mathrm{Xe}, \mathrm{Kr}$ ) for preplating has been explored at high temperatures, there are no studies at ultralow temperatures. Here the interest is that commensurate solids of different symmetries may be stabilized.

\section{Interacting Fermi Fluids}

The singular behaviour of correlated fermions in two dimensions has been the subject of intense theoretical controversy, dating from Anderson's conjecture that Landau Fermi liquids are destroyed in two dimensions [39]. ${ }^{3} \mathrm{He}$ films provide a variety of clean model 2D systems to test the validity of Landau Fermi liquid theory in 2D.

\subsection{Mott-Hubbard Transition}

The "second layer" of ${ }^{3} \mathrm{He}$ on graphite (with ${ }^{3} \mathrm{He}$ or ${ }^{4} \mathrm{He}$ first layer) forms an interacting 2D Fermi fluid, whose two-dimensional density can be tuned over a wide range. It therefore provides a model system for investigating interactions in strictly 2D and addressing the question whether Landau Fermi liquids exist in 2D. Although recent heat capacity studies show that ${ }^{3} \mathrm{He}$ condenses into a self-bound liquid at coverages less that $0.8 \mathrm{~nm}^{-2}$ [28], this still leaves a wide density range open. A ${ }^{3} \mathrm{He}$ monolayer on graphite plated by a bilayer of HD provides the clearest example of a densitydriven Mott transition [40]. There is a distinct effective mass divergence, while $F_{0}^{\mathrm{a}}$ depends only weakly on fluid density. This shows that helium is nearly localized, and not nearly ferromagnetic, as discussed in bulk ${ }^{3} \mathrm{He}$ [41]. The $2 \mathrm{D}$ solid that forms has antiferromagnetic exchange, just as bulk solid ${ }^{3} \mathrm{He}$ is antiferromagnetic. In the $2 \mathrm{D}$ case, it is a candidate quantum spin liquid. This supports the proposal that both antiferromagnetic and ferromagnetic spin fluctuations are important in ${ }^{3} \mathrm{He}$, and to the pairing interaction in the superfluid phases [42]. It seems that the fixed point of an antiferromagnetic Mott insulator controls the density-dependent Fermi liquid interactions. The experiment described above also determined that the beyond linear in $T$ term in the heat capacity is $T^{2}$. Microscopic theory also shows that Landau Fermi liquid survives in $2 \mathrm{D}$, but with non-analytic behaviour which determines this sub-leading term in the temperature dependence of the heat capacity [43].

Our recent NMR study [44] of the second layer of ${ }^{3} \mathrm{He}$ on graphite, preplated with a solid ${ }^{4} \mathrm{He}$ monolayer, shows a relatively wide density range over which there is a quantum coexistence of fluid and solid, with no evidence for a hole-doped Mott insulator 
and associated Fermi surface reconstruction. In this case, we argue that the ${ }^{3} \mathrm{He}$ experiences a density-tuned Wigner-Mott-Hubbard transition. The two cases probably differ because of the difference in strength of the periodic potential experienced by the atoms in the fluid layer, due to the different underlying layer $\left({ }^{4} \mathrm{He}\right.$ or $\left.\mathrm{HD}\right)$.

\subsection{D ${ }^{3} \mathrm{He}$ Built on Surface States on a ${ }^{4} \mathrm{He}$ Film}

Perhaps the most ideal substrate is the free surface of bulk ${ }^{4} \mathrm{He}$. As ${ }^{3} \mathrm{He}$ is added, it initially forms a 2D system through preferential binding to the free surface, and at higher ${ }^{3} \mathrm{He}$ content a 2D surface layer coexists with a 2D Fermi liquid in bulk.

A distinct system, and highly tuneable, is $2 \mathrm{D}{ }^{3} \mathrm{He}$ formed by populating the surface ground state on graphite plated with a discrete number of ${ }^{4} \mathrm{He}$ atomic layers. The behaviour is very sensitive to the number of ${ }^{4} \mathrm{He}$ layers. Such atomically layered films therefore present advantages over "helium mixture" films on a heterogeneous substrate, reviewed in $[45,46]$.

The simplest case studied so far is ${ }^{3} \mathrm{He}$ on four atomic layers of ${ }^{4} \mathrm{He}$ (two solid and two superfluid) [47]. Here the fermionic system is strictly 2D, in its ground state with respect to motion normal to surface and (importantly) with $2 \mathrm{D}$ interactions. As the density is tuned, the "fixed point" is no longer a Mott insulator, and the relative dependence of the Landau parameters $F_{0}^{\mathrm{a}}$ and $F_{1}^{\mathrm{s}}$ is quite different from that case, discussed previously. The Landau parameters are determined from high precision SQUID NMR over a wide temperature range to well below $1 \mathrm{mK}$ and heat capacity measurements [47, 48]. Analysis by Hartree-Fock theory shows that the 2D interactions are highly anisotropic, with strong backwards scattering; only s- and p-wave interactions are required. Again Fermi liquid theory survives. This is a clear result in an ideal 2D system. By contrast, in 2D cold atom systems the interactions are s-wave and three-dimensional (i.e. tuneable through a Feshbach resonance, which is absent in 2D) [49, 50].

The system of ${ }^{3} \mathrm{He}$ on four layers of ${ }^{4} \mathrm{He}$ is observed to exhibit condensation of 2D ${ }^{3} \mathrm{He}$ for coverages less than $0.3 \mathrm{~nm}^{-2}$. On the other hand, $2 \mathrm{D}^{3} \mathrm{He}$ on three layers of ${ }^{4} \mathrm{He}$ shows a series of instablities at ${ }^{3} \mathrm{He}$ coverages below $1 \mathrm{~nm}^{-2}$, detected through measurements of magnetic susceptibility [51]. For example, the coexistence of two Fermi fluids is observed, one of which has extremely low density (of order $0.04 \mathrm{~nm}^{-2}$ ). This system can be tuned to show a possible signature of ${ }^{3} \mathrm{He}$ dimer formation. Above ${ }^{3} \mathrm{He}$ coverage of $1 \mathrm{~nm}^{-2}$ a uniform $2 \mathrm{D}$ fermi fluid is recovered. Torsional oscillator measurements show that increasing the ${ }^{3} \mathrm{He}$ density drives a gradual suppression of superfluidity of the ${ }^{4} \mathrm{He}$ layer: a superfluid-insulator transition. The formation of a second Fermi fluid built on the first excited surface bound state, such as observed on a four-layer ${ }^{4} \mathrm{He}$ film [47], is now accompanied by strong ${ }^{3}$ He localization effects.

The message of these results is that the study of so-called helium mixture films on a graphite substrate benefits from the clear atomic layering. The coupling between the ${ }^{3} \mathrm{He}$ film and the ${ }^{4} \mathrm{He}$ "substrate" can even be strong enough to modify 
the state of the ${ }^{4} \mathrm{He}$ film. Clear confrontation between theory and experiment should be possible.

In the absence of such effects, where the focus is on an interacting Fermi system, this is a nice example of a coupled fermion-boson system [52]. ${ }^{3} \mathrm{He}-{ }^{3} \mathrm{He}$ interactions mediated by the ${ }^{4} \mathrm{He}$ film are enhanced by the presence of the substrate (so a uniform ${ }^{4} \mathrm{He}$ film thickness is crucial). The ${ }^{3} \mathrm{He}$ Fermi velocity is tuned by ${ }^{3} \mathrm{He}$ density, and the ${ }^{4} \mathrm{He}$ phonon/ripplon velocity is also tuned by ${ }^{3} \mathrm{He}$ density $[45,53]$. In principle, it may be possible (with an appropriate ${ }^{4} \mathrm{He}$ film) to tune through the point at which these velocities are equal. At this point, the usual separation of quasiparticle mass enhancement into a product of hydrodynamic (dynamic) mass and interaction terms breaks down $[54,55]$. This is of broad interest to the understanding of low-density 2D electronic systems [56, 57].

\section{Frustrated Magnetism}

In this section, we provide a brief overview of frustrated magnetism in two-dimensional solid ${ }^{3} \mathrm{He}$. The key contemporary challenge is the identification of a quantum spin liquid (QSL). In the following, we review the case for $2 \mathrm{D}$ solid ${ }^{3} \mathrm{He}$ as an ideal system to realize the QSL.

\subsection{Frustration by Atomic Ring Exchange}

The second layer of ${ }^{3} \mathrm{He}$ on graphite (plated by a monolayer of ${ }^{4} \mathrm{He}$ ) [58] or ${ }^{3} \mathrm{He}$ on graphite plated by a bilayer of HD [38] provides the cleanest examples of two-dimensional magnetism, in which exchange interactions dominate (dipolar spin-orbit interactions are negligible), and interlayer exchange couplings such as those present in quasi-2D solids are absent and with high tunability via adjustment of the ${ }^{3} \mathrm{He}$ coverage. The nuclear magnetism of $2 \mathrm{D}$ solid ${ }^{3} \mathrm{He}$ can be understood in terms of a model magnetic system in which frustration arises both from geometry (triangular lattice) and competing atomic ring exchange [59]. Ring exchange of an odd number of particles is ferromagnetic (FM), even is antiferromagnetic (AFM). The ring-exchange interactions are strong in $2 \mathrm{D}$ and significantly higher than in $3 \mathrm{D}$ solid helium, because of both high in-plane zero-point motion, low density and zeropoint motion out of plane. Thouless [60] first proposed the effective spin Hamiltonian, in terms of permutation operators:

$$
\begin{array}{ll}
\mathcal{H}=\sum_{n}(-1)^{n} J_{n} P_{n} & P_{2}=\frac{1}{2}\left(1+\sigma_{1} \cdot \sigma_{2}\right) \\
& P_{3}=\frac{1}{2}\left(1+\sigma_{1} \cdot \sigma_{2}+\sigma_{2} \cdot \sigma_{3}+\sigma_{3} \cdot \sigma_{1}\right) \\
& P_{4} \text { includes terms like }\left(\sigma_{1} \cdot \sigma_{2}\right)\left(\sigma_{3} \cdot \sigma_{4}\right) .
\end{array}
$$

The effective Heisenberg Hamiltonian $J=J_{2}-2 J_{3}$ is FM because three-particle exchange dominates two particle exchange. This is a consequence of the fact that helium atoms are "hard spheres" (Fig. 1). 
Fig. 1 Hierarchy of cyclic ringexchange interactions in $2 \mathrm{D}{ }^{3} \mathrm{He}$ on a triangular lattice $[59,61]$

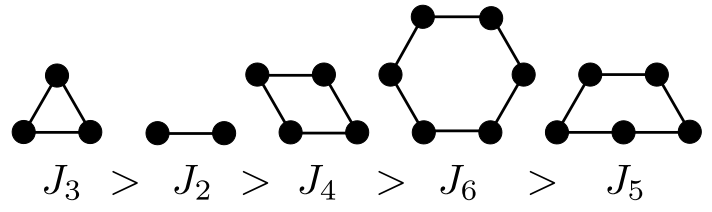

For simplicity, and for the purposes of illustration, we truncate at four-particle exchange. We refer to this two-parameter model as the $J-J_{4}$ model. In principle, these exchange parameters can be inferred from experiment, since the effective exchange parameters which enter the magnetic susceptibility, heat capacity and spin wave velocity to leading order are different and take the form:

$$
\begin{array}{rlrl}
\text { Curie-Weiss constant } & J_{\chi} & =-\left(J+3 J_{4}\right) & M=\frac{c}{T-\theta} \quad \theta=3 J_{\chi} \\
\text { Spin wave velocity } & J_{\mathrm{S}} & =-\left(J+4 J_{4}\right) & \\
\text { Heat capacity } & J_{c}^{2} & =\left(J+5 J_{4} / 2\right)^{2}+2 J_{4}^{2} & C=\frac{9}{4} N k_{\mathrm{B}}\left(\frac{J_{c}^{2}}{T^{2}}\right) .
\end{array}
$$

Combined measurements of heat capacity and magnetization (by NMR) on the same sample [62-64] demonstrate that indeed the leading-order temperature dependence is described by different exchange constants. Following the development of multiple spin exchange (MSE) high-temperature series expansions (HTSE) [65], these were used to analyse a body of heat capacity and magnetization data [61]. These results demonstrate that frustration by competing ring exchange persists into coverage régimes in which the magnetism shows a ferromagnetic tendency.

Studies of this frustrated ferromagnet, as a function of magnetic field by the broadband SQUID NMR method, show that it is an ideal 2D ferromagnet [66]. The Mermin-Wagner theorem is broken due to the small Zeeman gap in the spin wave spectrum. The low-temperature magnetism, which can be precisely determined from the dipolar frequency shift due to sample spin polarization, is well described by spin wave theory. Once again the frustrated spin exchange manifests through an inferred effective exchange constant for spin waves which differs from that determining hightemperature magnetism (Curie-Weiss constant).

The crossover from AFM to FM occurs in the vicinity of the third layer promotion. The question arises: What is the mechanism by which the relative strength of atomic ring-exchange interactions is tuned by total coverage? The interplay of the structure of the second layer and its magnetism as a function of total coverage has been extensively discussed [36]. This discussion will also be influenced by the result that the third layer self-condenses into 2D liquid puddles with a density of around $0.7 \mathrm{~nm}^{-2}$ [28]. Our unpublished work provides strong indications that RKKY interactions are an important contributor to FM exchange as proposed in [67, 68]. In this coverage régime in which the third layer fluid is puddled, our broadband SQUID NMR measurement indicates two contributions dominated by the localized second layer: an unshifted line, attributed to the AFM second layer with no fluid overlayer, and a shifted line arising from regions of the second layer with a puddle of the third 
layer fluid overlayer. Note that it has been shown the Mermin-Wagner theorem also holds for indirect RKKY-like exchange [69].

\subsection{Quantum Spin Liquid}

The quantum spin liquid is a highly entangled quantum ground state, yet to be conclusively realized in a physical system, and highly sought after in quasi-2D layered magnetic materials [70]. In ${ }^{3} \mathrm{He}$ films, the three candidate systems for the QSL are each a $2 \mathrm{D}$ solid monolayer of ${ }^{3} \mathrm{He}$ on a triangular lattice. This is a spin $\frac{1}{2}$ system $\left[{ }^{3} \mathrm{He}\right.$ nuclear spin]. The magnetization is directly, and selectively, measurable by NMR. In all cases, the putative QSL is at the border of a density-tuned Mott-Hubbard transition. As discussed, as well as the geometrical frustration of the triangular lattice, there is strong frustration due to competing atomic ring-exchange interactions. All these conditions are highly favourable for a QSL.

The candidate systems are as follows: (i) The second layer of ${ }^{3} \mathrm{He}$ on graphite, where the first layer is ${ }^{3} \mathrm{He}$. In this case, the first layer of ${ }^{3} \mathrm{He}$ is a compressed solid on a triangular lattice, as confirmed by neutron scattering [61], paramagnetic, with very weak exchange interaction with the second layer. The coupled magnetism of the first and second layer is a complication. However, the fact that the first layer is a weakly interacting "spectator" of the putative QSL in the second layer may prove to be advantageous. The heat capacity of this system has been measured to $100 \mu \mathrm{K}$ [71] and shows a double-peak structure which emerges in exact diagonalization studies of the $J, J_{4}$ model. (ii) A monolayer of ${ }^{3} \mathrm{He}$ on graphite, preplated by a solid monolayer of ${ }^{4} \mathrm{He}$. This system is very closely related to (i). However, the paramagnetic ${ }^{3} \mathrm{He}$ first layer is replaced with non-magnetic ${ }^{4} \mathrm{He}$. The density of the close-packed ${ }^{4} \mathrm{He}$ first layer triangular lattice is about $5 \%$ higher than the ${ }^{3} \mathrm{He}$ first layer. Given this close correspondence, we will refer to this system also as "the second layer of ${ }^{3} \mathrm{He}$ on graphite". (iii) A monolayer of ${ }^{3} \mathrm{He}$ on graphite, preplated by a solid bilayer of $\mathrm{HD}[38,72]$.

The high-temperature magnetism shows that the system has an antiferromagnetic character. Magnetization measurements into the microkelvin régime on both system (ii) and system (iii) support a gapless spin liquid [73]. In this latter experiment, measurements extended to $10 \mu \mathrm{K}$, and placed a bound of this order on the spin gap. In our recent work on system (ii), we find that the low-temperature magnetism is consistent with a Pauli susceptibility, as expected for a gapless spin liquid, with a characteristic energy scale of a few hundred $\mu \mathrm{K}$ [44].

We believe that systems (ii) and (iii) reflect a different balance between the periodic potential of the solid underlayer on a triangular lattice (HD bilayer or ${ }^{4} \mathrm{He}$ ) and intralayer ${ }^{3} \mathrm{He}$ interactions. The HD bilayer is of significantly lower density than the ${ }^{4} \mathrm{He}$ first layer, and the ${ }^{3} \mathrm{He}$ layer shows a Mott-Hubbard transition into a 4/7 or 7/12 triangular superlattice phase. The results for system (ii) are more consistent with a density wave instability in the ${ }^{3} \mathrm{He}$ layer. Theoretical simulations find that solid phase is stable at 7/12 relative density [74] (not 4/7 as in previous work [75]), but there is no evidence for the stability of this structure with respect to hole and interstitial doping as the density is varied around this value. This behaviour is indeed 
found in NMR studies in which the effective mass is inferred from fits of the magnetization to a solid plus Fermi fluid extending through a region of unconventional quantum coexistence [44]. There is no evidence for the appearance of a hole-doped Mott insulator on the low-density side, with associated Fermi surface reconstruction. This is suggestive of a Wigner-Mott transition. In this case of ${ }^{3} \mathrm{He}$ on ${ }^{4} \mathrm{He}$, the $7 / 12$ phase occurs very close to the third layer promotion.

In system (iii), exchange in the Mott insulator is much stronger than in system (ii) $[38,76]$. This is understood in terms of the lower density. Therefore, a monolayer of ${ }^{3} \mathrm{He}$ on graphite preplated by a solid bilayer of HD may be the most promising for demonstrating quantum spin liquid behaviour.

Thus, 2D ${ }^{3} \mathrm{He}$ offers a persuasive candidate to realize a gapless QSL. Although it exists in a challenging temperature régime, we have a powerful tool to probe it: $\mathrm{NMR}$ on the ${ }^{3} \mathrm{He}$ spin. A future experimental challenge is to conclusively identify the QSL ground state and demonstrate its quantum entanglement. This might include: unambiguous measurement of the heat capacity to identify predicted nonFermi liquid behaviour as signature of the emergent gauge field [77]; thermal transport by spinons; investigation of spin dynamics, such as spin-lattice relaxation time.

As far as theory is concerned, it is to be hoped that increased computational power will lead to improvements in comparison between MSE theory and experiment. Currently, the HTSE go to only fifth order [65], whereas the Heisenberg model goes to thirteenth order [78]. HTSE used in conjunction with Padé approximants is a powerful tool to analyse thermodynamic properties, see [61, 63]. The MSE parameters are the essential input for finite size exact diagonalization studies [79], which predict both the ground state and the evolution of magnetization with applied magnetic field [79, 80]. In the latter case, the key observables are plateaux in the magnetization as a function of field, and the field at which saturation magnetization is observed. According to [80], the data of [81] are, for a particular choice of MSE parameters, consistent with a spin nematic ground state. Refinement in the precision of MSE parameters in conjunction with numerical theory exploiting improved computational power is desirable. However, while the utility of the MSE model to describe the experimental data, albeit with several exchange parameters, cannot be denied, it is probably worth exercising caution when trying to account for the highly entangled QSL state. See critique of [82] in the context of bulk ${ }^{3} \mathrm{He}$. An alternative point of view is that the essential ingredient to establish a quantum spin liquid in the case of $2 \mathrm{D}{ }^{3} \mathrm{He}$ is charge fluctuations [83, 84], either at the border of a Mott transition, or possibly (in the case of the "second layer") because of proximity to the third layer promotion.

\section{$6{ }^{3} \mathrm{He}$ Heavy Fermion Quantum Criticality}

A ${ }^{3} \mathrm{He}$ bilayer grown on graphite plated by a bilayer of solid ${ }^{4} \mathrm{He}$ was found to behave as a heavy fermion system with quantum criticality [85]. It appears to fall into the class of orbital-selective Mott transition [86], with a Kondo breakdown QCP [87-91]. The lower ${ }^{3} \mathrm{He}$ layer (L1) plays the role of the f-fermions, and the second layer (L2) is analogous to the mobile conduction electrons. The solid ${ }^{4} \mathrm{He}$ 
bilayer preplating creates a composite substrate in which L1 remains fluid as the second layer L 2 forms. The two layers are hybridized by a Kondo interaction: in this case exchange of atoms between the two layers. This is tuned by the density of the upper layer. A maximum in both heat capacity and magnetization, which track to lower temperatures with increasing coverage, identifies the coherence temperature below which the heavy fermion state of the coupled bilayer is formed. A densitytuned quantum critical point (QCP) is found at which the effective mass diverges. Beyond this QCP, layer L1 is localized and layer L2 is itinerant, consisting of weakly interacting $2 \mathrm{D}$ fermions. The frustrated magnetism of atomic ring exchange plays a role in L1. Approach to the QCP is intercepted by a magnetic instability, which it is believed is triggered when the ferromagnetic exchange in L1 dominates the interlayer Kondo coupling [92, 93]. Following the prediction by [89], it was found that the Curie-Weiss temperature measured above the coherence temperature is zero at this instability coverage. Quantum criticality in the ${ }^{3} \mathrm{He}$ heavy fermion bilayer provides a simple system to further understanding of the interplay between low dimensionality, frustrated magnetism and Kondo breakdown-induced Fermi surface reconstruction.

\section{Two-Dimensional Supersolid}

The identification of a supersolid state of matter has excited interest across the broad spectrum of the quantum fluids and solids community, and the cold atomic gases community. In principle, one way a solid (the key property of which is rigidity) can exhibit superfluidity is if solid and superfluid orders coexist. Mechanisms include mobile zero-point vacancies within a solid structure or superfluidity in dislocation cores. Reviews of supersolid ${ }^{4} \mathrm{He}$ include [94-97]. Such systems necessarily feature small superfluid fractions, and detection of any superfluid response requires it to be disentangled from viscoelastic response [98]. Recently, evidence for the engineering of a "supersolid" in cold atoms with long-range dipolar interactions has been reported. In this case, the system can be tuned into a periodic structure of superfluid droplets with phase coherence across the droplet array [99-102].

Evidence for an emergent two-dimensional supersolid in the second layer of ${ }^{4} \mathrm{He}$ on graphite is reported in [103, 104]. This work was motivated by the detection in earlier torsional oscillator experiments on this system, which found an anomalous mass decoupling over a narrow coverage range [24]. In that work, the destruction of superfluidity with increasing coverage was attributed to solidification of the film. The recent torsional oscillator study $[103,104]$ was made over a fine grid of coverages down to temperatures approaching $1 \mathrm{mK}$. The results led to the proposal of a state of intertwined density wave and superfluid order. The intertwined state, in which the two seemingly incompatible orders are entangled, can explain the enigma of supersolidity and the large superfluid fraction observed. It was suggested that the enlarged symmetry typical of such intertwined states [105] accounts for the absence of a BKT transition, since vortices are no longer stable defects. In the second layer supersolid, the anomalous temperature dependence of the superfluid density in the 
low-temperature limit was explained in terms of a spectrum of elementary excitations with a set of softening roton minima. It follows that the structure factor is strongly peaked at the momenta of these minima: density wave order. A sequence of four coverages intervals with distinct features of data collapse, two with single parameter scaling and two with two-parameter scaling, provided further evidence of the interplay between film structure and superfluid response.

Independent evidence for the formation of a low-temperature ordered phase, well aligned with the observed supersolid phase, comes from the coverage dependence of heat capacity anomalies at $1-1.5 \mathrm{~K}[13,106,107]$. This is in stark contrast to theoretical simulations which find no solid phase at the densities at which both supersolid response and melting signatures are observed [108-112].

One approach to probe the structure of the second layer at ultralow temperatures is to dope the ${ }^{4} \mathrm{He}$ layer with a small concentration of ${ }^{3} \mathrm{He}$ and rely on different thermodynamic properties of fluid or localized ${ }^{3} \mathrm{He}$ phases, to infer the state of the host ${ }^{4} \mathrm{He}$ film. Heat capacity and NMR measurements clearly confirm that the film enters a solid phase in this density range (contradicting the first principles simulations) [113].

However, there is a number of subtle and interesting features. These derive from the fact that in a quantum solid the atoms are mobile: this leads to delocalized ${ }^{3} \mathrm{He}$ impuritons (quasiparticle excitations) in dilute bulk mixtures [114, 115]. However, in the $2 \mathrm{D}$ case, exchange rates and hence the tunnelling bandwidth are large, so that attractive strain-mediated interactions between ${ }^{3} \mathrm{He}$ impurities can be overcome and the conditions for quantum degeneracy realized. The localization of ${ }^{3} \mathrm{He}$ impurities is the subject of ongoing work, including studies of the ${ }^{3} \mathrm{He}$ spin-lattice relaxation time. These show a remarkable and sharp increase in $T_{1}$ with onset at low $T$ that is particularly pronounced near 4/7 (7/12) superlattice densities. This phenomenon may be related to many-body localization [116].

These results support the conclusion that the novel superfluid responses reported in $[103,104]$ occur in a 2D solid phase. The following further work is desirable: to check the frequency independence of the supersolid response; to detect the response on a higher quality substrate; to understand the collective mode spectrum of the putative intertwined state; to seek an underlying microscopic theory which gives rise to this state.

\section{Superfluid ${ }^{3} \mathrm{He}$ Films}

The superfluidity of thin atomically layered ${ }^{3} \mathrm{He}$ films has so far eluded observation. The strictly $2 \mathrm{D}$ limit corresponds to $k_{\mathrm{F}}^{-1} \sim D \ll \xi_{0}$, where $\xi_{0}=h \nu_{\mathrm{F}} / 2 \pi k_{\mathrm{B}} T_{\mathrm{c}}$ is the zero temperature coherence length and $D$ is the film thickness. The $2 \mathrm{D}$ superfluidity of a ${ }^{3} \mathrm{He}$ monolayer has been discussed theoretically in [117-122]. In the case of p-wave pairing, this will be sensitive to non-magnetic disorder and requires high-quality substrates. The pairing mechanism is likely to be highly dependent on the composite substrate for the 2D Fermi system. Thus, ${ }^{3} \mathrm{He}$ on a superfluid ${ }^{4} \mathrm{He}$ "substrate" can interact via ${ }^{4} \mathrm{He}$ surface phonon/ripplon excitations. It is also worth noting that while pairing via exchange of spin fluctuations plays an important role in 
bulk superfluid ${ }^{3} \mathrm{He}$, the spectrum of spin fluctuations will differ in 2D. In all cases, the "bottom-up" growth of suitable ${ }^{3} \mathrm{He}$ films requires high-quality substrates.

How to approach the strictly 2D limit in a controlled way? While in superfluid ${ }^{4} \mathrm{He}$ the coherence length is of atomic scale, in bulk superfluid ${ }^{3} \mathrm{He}$ the diameter of the Cooper pair $\xi_{0}$ at zero pressure is around $80 \mathrm{~nm}$. For a surface for which ${ }^{3} \mathrm{He}$ quasiparticle scattering is diffuse, superfluidity of the film is suppressed for films thinner than this. Stabilization of van der Waals films of such thickness is tricky in the face of competing effects of surface tension and gravity. A specular surface can be created by depositing a superfluid ${ }^{4} \mathrm{He}$ film. Perhaps, the ultimately smooth surface is that of bulk superfluid ${ }^{4} \mathrm{He}$. In this case, a 2D ${ }^{3} \mathrm{He}$ surface film can coexist with a bulk dilute solution. This potential of this system has been emphasized in [123], where it has been studied by a Wigner crystal of electrons on the surface. However, these create a regular surface deformation commensurate with the electron density. Nevertheless, subject to appropriate developments in technique, this surface could also be probed ultrasonically, or potentially by NMR.

In contrast with such "self-assembled" films, a different approach adopted recently is to use nanofabrication methods to define a cavity, in the simplest case creating a thin slab geometry, into which helium is admitted through a fill line. This can be thought of as a film, of thickness precisely defined by the height of the cavity, with equivalent upper and lower surfaces. This strategy is particularly suitable for the study of topological superfluid ${ }^{3} \mathrm{He}$ in the quasi-2D limit, $k_{\mathrm{F}}^{-1} \ll D<10 \xi_{0}$. So far cavities of height $D$ in the range 1000-100 nm have been studied [124-128]. An advantage is that for fixed cavity height the effective confinement $\xi_{0} / D$ is tuneable by pressure, since $\xi_{0}=h \nu_{\mathrm{F}} / 2 \pi k_{\mathrm{B}} T_{\mathrm{c}}$.

This limit is distinct from the strictly $2 \mathrm{D}$ limit, since in such cavities the normal Fermi liquid is 3D. However, given that specular surfaces are achievable by coating with a superfluid ${ }^{4} \mathrm{He}$ film, the film thickness (cavity height) can be shrunk towards the $2 \mathrm{D}$ limit. Then, size quantization along $\mathbf{z}$ plays a role and the Fermi sphere breaks up into Fermi discs, where the number of $2 \mathrm{D}$ mini-bands is $j=k_{\mathrm{F}} D / \pi$. This opens up a wealth of new quantum states, associated with the integer number of bands, which in principle can be tuned by slab thickness [129]. Size quantization effects have already been seen in measurement of momentum relaxation in the flow of an unsaturated normal ${ }^{3} \mathrm{He}$ film over a polished silver surface with fully characterized surface roughness $[130,131]$. In this case, the picture is that the quasi-2D mini-bands are subject to an effective disorder potential $v(x, y)$ that is determined by the fluctuations in confining cavity height $D+d(x, y)$, due to surface roughness or longer length scale variations in cavity height $[132,133]$. Since variations in cavity height can be measured, at least in principle, we have the unusual situation of a disorder potential that can be fully determined experimentally.

\section{Future Prospects}

A future quest is for new graphite-based substrates of improved quality relative to exfoliated graphite. Obvious candidates are: graphene (including multilayer graphene) and carbon nanotubes. Theoretically, the strong similarities between the 
growth of helium on graphene and graphite are established. Experimentally, there are multiple issues: contamination of the graphene surface and requirement for new measurement techniques tailored to measurements on samples with small surface area. The growth of helium films on a nanotube operated as a nanomechanical resonator has recently been demonstrated, with evidence of first-order layering transitions which testify to substrate quality [134]. Elsewhere the sensitivity of electrical transport through a carbon nanotube to a variety of adsorbates, including helium, has been demonstrated [135-137]. The commercial availability of large area graphene grown by CVD also offers opportunities. Attention has also been drawn to future opportunities in the study of monolayer films on graphene-derived substrates, such as graphane and fluorographene, with new phenomena such as anisotropic effects in sub-monolayer films [138]. Again progress is subject both to the ability to create pristine surfaces and to development of measurement techniques of adequate sensitivity.

The helium isotopes in condensed form are unique, and our ability to fashion them into a wide range of quantum materials is continuously developing. This demands the development of new techniques, exploitation of new generations of quantum sensors and the pursuit of experiments yet further into the microkelvin régime. ${ }^{4} \mathrm{He}$ and ${ }^{3} \mathrm{He}$ have supplied a wide range of paradigms in the past, and there is no sign of exhaustion in this seam of enquiry.

Acknowledgements Experiments at Royal Holloway on atomically layered films have been a result of collaborations, taken chronologically, with: Chris Lusher, Marcio Siqueira, Martin Dann, Bob Ray, Andrew Casey, Hetal Patel, Michael Neumann, Lev Levitin, Anastasia Phillis, Frank Arnold, Ben Yager, Kristian Kent, Alexander Waterworth, Jan Knapp. Collaboration with Jeevak Parpia, Chris Howard, Thomas Schurig, Dietmar Drung is gratefully acknowledged. Theoretical input from Michel Roger, Bernard Bernu, Gregoir Misguich, Catherine Pépin, Fakher Assaad, Piers Coleman, Andrew Ho, Derek Lee, Andrej Chubukov was particularly important and is gratefully acknowledged, as well as discussions with members of the European Microkelvin Platform. We also thank Henri Godfrin, Eddy Collin and Hiroshi Fukuyama and their co-workers for discussions. Our research on atomically layered films was most recently supported by EPSRC (UK) through EP/H048375/1, and the European Union's Horizon 2020 Research and Innovation Programme, under Grant Agreement No. 824109 (European Microkelvin Platform).

Open Access This article is licensed under a Creative Commons Attribution 4.0 International License, which permits use, sharing, adaptation, distribution and reproduction in any medium or format, as long as you give appropriate credit to the original author(s) and the source, provide a link to the Creative Commons licence, and indicate if changes were made. The images or other third party material in this article are included in the article's Creative Commons licence, unless indicated otherwise in a credit line to the material. If material is not included in the article's Creative Commons licence and your intended use is not permitted by statutory regulation or exceeds the permitted use, you will need to obtain permission directly from the copyright holder. To view a copy of this licence, visit http://creativecommons.org/licen ses/by/4.0/.

\section{References}

1. A. Thomy, X. Duval, J. Chim. Phys. 66, 1966 (1969). https://doi.org/10.1051/jcp/196966s21966

2. A. Thomy, X. Duval, J. Chim. Phys. 67, 286 (1970). https://doi.org/10.1051/jcp/1970670286

3. A. Thomy, X. Duval, J. Chim. Phys. 67, 1101 (1970). https://doi.org/10.1051/jcp/1970671101

4. J.G. Dash, Films on Solid Surfaces (Academic Press, New York, 1975) 
5. J.G. Dash, J. Ruvalds, Phase Transitions in Surface Films (Plenum Press, New York, 1980)

6. H. Taub, G. Torzo, H.J. Lauter, J.S.C. Fain, Phase Transitions in Surface Films 2 (Plenum Press, New York, 1991)

7. R.J. Birgeneau, P.M. Horn, Science 232(4748), 329 (1986). https://doi.org/10.1126/scien ce.232.4748.329

8. M. Bretz, Phys. Rev. Lett. 38(9), 501 (1977). https://doi.org/10.1103/PhysRevLett.38.501

9. S. Nakamura, K. Matsui, T. Matsui, H. Fukuyama, J. Low Temp. Phys. 171(5-6), 711 (2013). https ://doi.org/10.1007/s10909-012-0847-5

10. B.P. Cowan, M.G. Richards, A.L. Thomson, W.J. Mullin, Phys. Rev. Lett. 34, 165 (1977). https:// doi.org/10.1103/PhysRevLett.38.165

11. B. Cowan, L. Abou El-Nasr, M. Fardis, A. Hussain, Phys. Rev. Lett. 58(22), 2308 (1987). https:// doi.org/10.1103/PhysRevLett.58.2308

12. S.V. Hering, S.W. Van Sciver, O.E. Vilches, J. Low Temp. Phys. 25(5-6), 793 (1976). https://doi. org/10.1007/bf00657299

13. D.S. Greywall, Phys. Rev. B 47(1), 309 (1993). https://doi.org/10.1103/PhysRevB.47.309

14. L.W. Bruch, M.W. Cole, E. Zaremba, Physical Adsorption: Forces and Phenomena (Clarenden Press, Oxford, 1997)

15. H. Franco, R.E. Rapp, H. Godfrin, Phys. Rev. Lett. 57(9), 1161 (1986). https://doi.org/10.1103/ PhysRevLett.57.1161

16. H. Godfrin, H.J. Lauter, Prog. Low Temp. Phys. XIV 213 (1995)

17. A.I. Ahonen, T.A. Alvesalo, T. Haavasoja, M.C. Veuro, Phys. Rev. Lett. 41(7), 494 (1978). https:// doi.org/10.1103/PhysRevLett.41.494

18. H.M. Bozler, T. Bartolac, K. Luey, A.L. Thomson, Phys. Rev. Lett. 41(7), 490 (1978). https://doi. org/10.1103/PhysRevLett.41.490

19. L.J. Friedman, A.L. Thomson, C.M. Gould, H.M. Bozler, P.B. Weichman, M.C. Cross, Phys. Rev. Lett. 62(14), 1635 (1989). https://doi.org/10.1103/PhysRevLett.62.1635

20. A. Yamaguchi, T. Watanuki, R. Masutomi, H. Ishimoto, Phys. Rev. Lett. 93(16), 165301 (2004). https://doi.org/10.1103/PhysRevLett.93.165301

21. D. Bishop, J. Reppy, Phys. Rev. Lett. 40, 1727 (1978). https://doi.org/10.1103/PhysRevLet t. 40.1727

22. V.L. Berezinskii, Soviet Physics - JETP 34(3), 610 (1972). 1972JETP...34.610B

23. J.M. Kosterlitz, D.J. Thouless, J. Phys. C: Solid State Phys. 6(7), 1181 (1973). https://doi. org/10.1088/0022-3719/6/7/010

24. P.A. Crowell, J.D. Reppy, Phys. Rev. B 53(5), 2701 (1996). https://doi.org/10.1103/PhysR evB.53.2701

25. J. Nyéki, R. Ray, G. Sheshin, V. Maidanov, V. Mikheev, B. Cowan, J. Saunders, Low Temp. Phys. (USSR) 23(5), 379 (1997). https://doi.org/10.1063/1.593382

26. J. Nyéki, R. Ray, B. Cowan, J. Saunders, Phys. Rev. Lett. 81(1), 152 (1998). https://doi. org/10.1103/PhysRevLett.81.152

27. J. Saunders, C.P. Lusher, B.P. Cowan, Phys. Rev. Lett. 64(21), 2523 (1990). https://doi.org/10.1103/ PhysRevLett.64.2523

28. D. Sato, K. Naruse, T. Matsui, H. Fukuyama, Phys. Rev. Lett. 109(23), 235306 (2012). https://doi. org/10.1103/PhysRevLett.109.235306

29. R.E. Rapp, H. Godfrin, Phys. Rev. B 47(18), 12004 (1993). https://doi.org/10.1103/physr evb.47.12004

30. H. Ikegami, K. Obara, D. Ito, H. Ishimoto, Phys. Rev. Lett. 81(12), 2478 (1998). https://doi. org/10.1103/PhysRevLett.81.2478

31. D.S. Greywall, P.A. Busch, Phys. Rev. Lett. 65(22), 2788 (1990). https://doi.org/10.1103/PhysR evLett.65.2788

32. M. Morishita, H. Nagatani, H. Fukuyama, Phys. Rev. B 65, 10 (2002). https://doi.org/10.1103/ PhysRevB.65.104524

33. G. Zimmerli, G. Mistura, M.H. Chan, Phys. Rev. Lett. 68(1), 60 (1992). https://doi.org/10.1103/ PhysRevLett.68.60

34. B.E. Clements, E. Krotscheck, H.J. Lauter, Phys. Rev. Lett. 70(9), 1287 (1993). https://doi. org/10.1103/PhysRevLett.70.1287

35. B.E. Clements, J.L. Epstein, E. Krotscheck, M. Saarela, Phys. Rev. B 48(10), 7450 (1993). https:// doi.org/10.1103/physrevb.48.7450

36. H. Fukuyama, J. Phys. Soc. Jpn. 77(11), 111013 (2008). https://doi.org/10.1143/jpsj.77.111013 
37. C.P. Lusher, J. Saunders, B.P. Cowan, Phys. B: Cond. Matter. 165-166, 691 (1990). https://doi. org/10.1016/s0921-4526(90)81195-t

38. M. Siqueira, C.P. Lusher, B.P. Cowan, J. Saunders, Phys. Rev. Lett. 71(9), 1407 (1993). https://doi. org/10.1103/PhysRevLett.71.1407

39. P.W. Anderson, Phys. Rev. Lett. 65(18), 2306 (1990). https://doi.org/10.1103/PhysRevLett.65.2306

40. A. Casey, H. Patel, J. Nyéki, B.P. Cowan, J. Saunders, Phys. Rev. Lett. 90(11), 115301 (2003). https://doi.org/10.1103/PhysRevLett.90.115301

41. D. Vollhardt, Rev. Mod. Phys. 56(1), 99 (1984). https://doi.org/10.1103/RevModPhys.56.99

42. J.J. Wiman, J.A. Sauls (in preparation)

43. A.V. Chubukov, D.L. Maslov, S. Gangadharaiah, L.I. Glazman, Phys. Rev. Lett. 95(2), 026402 (2005). https://doi.org/10.1103/PhysRevLett.95.026402

44. F. Arnold, J. Nyéki, B. Cowan, J. Saunders (in preparation)

45. R. Hallock, Prog. Low Temp. Phys. XIV 321, (1995)

46. R.B. Hallock, J. Low Temp. Phys. 121(5/6), 441 (2000). https://doi.org/10.1023/a:1017514100122

47. M. Dann, J. Nyéki, B.P. Cowan, J. Saunders, Phys. Rev. Lett. 82(20), 4030 (1999). https://doi. org/10.1103/PhysRevLett.82.4030

48. A. Waterworth, J. Nyéki, B. Cowan, J. Saunders (in preparation)

49. A.T. Sommer, L.W. Cheuk, M.J. Ku, W.S. Bakr, M.W. Zwierlein, Phys. Rev. Lett. 108(4), 045302 (2012). https://doi.org/10.1103/PhysRevLett.108.045302

50. M. Feld, B. Frohlich, E. Vogt, M. Koschorreck, M. Kohl, Nature 480(7375), 75 (2011). https://doi. org/10.1038/nature 10627

51. A. Waterworth, J. Nyéki, B. Cowan, J. Saunders (in preparation)

52. N. Yokoshi, S. Kurihara, Phys. Rev. B 68, 6 (2003). https://doi.org/10.1103/PhysRevB.68.064501

53. E. Krotscheck, M.D. Miller, Phys. Rev. B 73, 13 (2006). https://doi.org/10.1103/PhysR evB.73.134514

54. A.J. Leggett, Ann. Phys. NY 46(1), 76 (1968). https://doi.org/10.1016/0003-4916(68)90304-7

55. V. Chubukov, A. Klein, D.L. Maslov, J. Exp. Theor. Phys. 127(5), 826 (2018). https://doi. org/10.1134/S1063776118110122

56. S. Qin, J. Kim, Q. Niu, C.K. Shih, Science 324(5932), 1314 (2009). https://doi.org/10.1126/scien ce. 1170775

57. J.F. Ge, Z.L. Liu, C. Liu, C.L. Gao, D. Qian, Q.K. Xue, Y. Liu, J.F. Jia, Nat. Mater. 14(3), 285 (2015). https://doi.org/10.1038/nmat4153

58. C.P. Lusher, J. Saunders, B.P. Cowan, Europhys. Lett. (EPL) 14(8), 809 (1991). https://doi. org/10.1209/0295-5075/14/8/015

59. M. Roger, Phys. Rev. Lett. 64(3), 297 (1990). https://doi.org/10.1103/PhysRevLett.64.297

60. D.J. Thouless, Proc. Phys. Soc. 86(5), 893 (1965). https://doi.org/10.1088/0370-1328/86/5/301

61. M. Roger, C. Bäuerle, Y.M. Bunkov, A.S. Chen, H. Godfrin, Phys. Rev. Lett. 80(6), 1308 (1998). https://doi.org/10.1103/PhysRevLett.80.1308

62. M. Siqueira, J. Nyéki, B. Cowan, J. Saunders, Czech. J. Phys. 46(S6), 3033 (1996). https://doi. org/10.1007/bf02548107

63. M. Siqueira, J. Nyéki, B. Cowan, J. Saunders, Phys. Rev. Lett. 76(11), 1884 (1996). https://doi. org/10.1103/PhysRevLett.76.1884

64. M. Siqueira, J. Nyéki, B. Cowan, J. Saunders, Phys. Rev. Lett. 78(13), 2600 (1997). https://doi. org/10.1103/PhysRevLett.78.2600

65. M. Roger, Phys. Rev. B 56(6), R2928 (1997). https://doi.org/10.1103/PhysRevB.56.R2928

66. A. Casey, M. Neumann, B. Cowan, J. Saunders, N. Shannon, Phys. Rev. Lett. 111(12), 125302 (2013). https://doi.org/10.1103/PhysRevLett.111.125302

67. R.A. Guyer, Phys. Rev. Lett. 64(16), 1919 (1990). https://doi.org/10.1103/PhysRevLett.64.1919

68. S. Tasaki, Prog. Theor. Phys. 79(6), 1311 (1988). https://doi.org/10.1143/ptp.79.1311

69. D. Loss, F.L. Pedrocchi, A.J. Leggett, Phys. Rev. Lett. 107(10), 107201 (2011). https://doi. org/10.1103/PhysRevLett.107.107201

70. L. Savary, L. Balents, Rep. Prog. Phys. 80(1), 016502 (2017). https://doi.org/10.1088/00344885/80/1/016502

71. K. Ishida, M. Morishita, K. Yawata, H. Fukuyama, Phys. Rev. Lett. 79(18), 3451 (1997). https:// doi.org/10.1103/PhysRevLett.79.3451

72. A. Casey, H. Patel, J. Nyéki, B.P. Cowan, J. Saunders, J. Low Temp. Phys. 113(3/4), 265 (1998). https://doi.org/10.1023/a:1022590331361 
73. R. Masutomi, Y. Karaki, H. Ishimoto, Phys. Rev. Lett. 92(2), 025301 (2004). https://doi. org/10.1103/PhysRevLett.92.025301

74. M.C. Gordillo, J. Boronat, Phys. Rev. B 94, 16 (2016). https://doi.org/10.1103/PhysRevB.94.16542 1

75. T. Takagi, J. Phys. Conf. Ser. 150(3), 032102 (2009). https://doi.org/10.1088/1742$6596 / 150 / 3 / 032102$

76. H. Ikegami, R. Masutomi, K. Obara, H. Ishimoto, Phys. Rev. Lett. 85(24), 5146 (2000). https://doi. org/10.1103/PhysRevLett.85.5146

77. O.I. Motrunich, Phys. Rev. B 72, 4 (2005). https://doi.org/10.1103/PhysRevB.72.045105

78. N. Elstner, R.R. Singh, A.P. Young, Phys. Rev. Lett. 71(10), 1629 (1993). https://doi.org/10.1103/ PhysRevLett.71.1629

79. G. Misguich, B. Bernu, C. Lhuillier, J. Low Temp. Phys. 110(1/2), 327 (1998). https://doi. org/10.1023/a:1022588817636

80. T. Momoi, P. Sindzingre, K. Kubo, Phys. Rev. Lett. 108(5), 057206 (2012). https://doi.org/10.1103/ PhysRevLett.108.057206

81. H. Nema, A. Yamaguchi, T. Hayakawa, H. Ishimoto, Phys. Rev. Lett. 102(7), 075301 (2009). https ://doi.org/10.1103/PhysRevLett.102.075301

82. M.C. Cross, D.S. Fisher, Rev. Mod. Phys. 57(4), 881 (1985). https://doi.org/10.1103/RevModPhys .57 .881

83. T. Mizusaki, M. Imada, Phys. Rev. B 74, 1 (2006). https://doi.org/10.1103/PhysRevB.74.014421

84. S. Watanabe, M. Imada, J. Phys. Soc. Jpn. 76(11), 113603 (2007). https://doi.org/10.1143/ jpsj.76.113603

85. M. Neumann, J. Nyéki, B. Cowan, J. Saunders, Science 317(5843), 1356 (2007). https://doi. org/10.1126/science.1143607

86. M. Vojta, J. Low Temp. Phys. 161(1-2), 203 (2010). https://doi.org/10.1007/s10909-010-0206-3

87. A. Benlagra, C. Pépin, Phys. Rev. Lett. 100(17), 176401 (2008). https://doi.org/10.1103/PhysR evLett.100.176401

88. C. Pépin, Phys. Rev. B 77, 24 (2008). https://doi.org/10.1103/PhysRevB.77.245129

89. A. Rançon-Schweiger, A. Benlagra, C. Pépin, Phys. Rev. B 83, 7 (2011). https://doi.org/10.1103/ PhysRevB.83.073102

90. K.S.D. Beach, F.F. Assaad, Phys. Rev. B 83, 4 (2011). https://doi.org/10.1103/PhysRevB.83.04510 3

91. S. Sen, N.S. Vidhyadhiraja, Phys. Rev. B 93, 15 (2016). https://doi.org/10.1103/PhysR evB.93.155136

92. N. Neumann, J. Nyéki, L. Levitin, A. Casey, B. Cowan, J. Saunders (in preparation)

93. J. Werner, F.F. Assaad, Phys. Rev. B 90, 20 (2014). https://doi.org/10.1103/PhysRevB.90.205122

94. L. Pollet, M. Boninsegni, A.B. Kuklov, N.V. Prokof'ev, B.V. Svistunov, M. Troyer, Phys. Rev. Lett. 98(13), 135301 (2007). https://doi.org/10.1103/PhysRevLett.98.135301

95. M. Boninsegni, N.V. Prokof'ev, Rev. Mod. Phys. 84(2), 759 (2012). https://doi.org/10.1103/RevMo dPhys.84.759

96. M.H.W. Chan, R.B. Hallock, L. Reatto, J. Low Temp. Phys. 172(5-6), 317 (2013). https://doi. org/10.1007/s10909-013-0882-X

97. R. Hallock, Phys. Today 68(5), 30 (2015). https://doi.org/10.1063/pt.3.2782

98. J. Beamish, J. Low Temp. Phys. 197(3), 187 (2019). https://doi.org/10.1007/s10909-019-02231-5

99. L. Tanzi, E. Lucioni, F. Fama, J. Catani, A. Fioretti, C. Gabbanini, R.N. Bisset, L. Santos, G. Modugno, Phys. Rev. Lett. 122(13), 130405 (2019). https://doi.org/10.1103/PhysRevLet t.122.130405

100. L. Chomaz, D. Petter, P. Ilzhöfer, G. Natale, A. Trautmann, C. Politi, G. Durastante, R. van Bijnen, A. Patscheider, M. Sohmen, M. Mark, F. Ferlaino, Phys. Rev. X 9, 2 (2019). https://doi. org/10.1103/PhysRevX.9.021012

101. F. Böttcher, J.N. Schmidt, M. Wenzel, J. Hertkorn, M. Guo, T. Langen, T. Pfau, Phys. Rev. X 9, 1 (2019). https://doi.org/10.1103/PhysRevX.9.011051

102. L. Tanzi, S.M. Roccuzzo, E. Lucioni, F. Fama, A. Fioretti, C. Gabbanini, G. Modugno, A. Recati, S. Stringari, Nature (2019). https://doi.org/10.1038/s41586-019-1568-6

103. J. Nyéki, A. Phillis, A. Ho, D. Lee, P. Coleman, J. Parpia, B. Cowan, J. Saunders, Nat. Phys. 13(5), 455. https://doi.org/10.1038/nphys4023

104. J. Nyéki, A. Phillis, B. Cowan, J. Saunders, J. Low Temp. Phys. 187(5-6), 475 (2017). https://doi. org/10.1007/s10909-017-1779-X 
105. E. Fradkin, S.A. Kivelson, J.M. Tranquada, Rev. Mod. Phys. 87(2), 457 (2015). https://doi. org/10.1103/RevModPhys.87.457

106. D.S. Greywall, P.A. Busch, Phys. Rev. Lett. 67(25), 3535 (1991). https://doi.org/10.1103/PhysR evLett.67.3535

107. S. Nakamura, K. Matsui, T. Matsui, H. Fukuyama, Phys. Rev. B 94, 18 (2016). https://doi. org/10.1103/PhysRevB.94.180501

108. P. Corboz, M. Boninsegni, L. Pollet, M. Troyer, Phys. Rev. B 78(24), 245414 (2008). https://doi. org/10.1103/PhysRevB.78.245414

109. M.C. Gordillo, J. Boronat, Phys. Rev. B 85, 19 (2012). https://doi.org/10.1103/PhysRevB.85.19545 7

110. J. Ahn, H. Lee, Y. Kwon, Phys. Rev. B 93, 6 (2016). https://doi.org/10.1103/PhysRevB.93.064511

111. Y. Kwon, D.M. Ceperley, Phys. Rev. B 85, 22 (2012). https://doi.org/10.1103/PhysRevB.85.22450 1

112. J. Happacher, P. Corboz, M. Boninsegni, L. Pollet, Phys. Rev. B 87, 9 (2013). https://doi. org/10.1103/PhysRevB.87.094514

113. J. Knapp, J. Nyeki, B. Cowan, J. Saunders (in preparation)

114. A.F. Andreev, I. Lifshitz, JETP (USSR) 29, 1107 (1969)

115. A.F. Andreev, Prog. Low Temp. Phys. VIII 67 (1982)

116. R. Nandkishore, D.A. Huse, Annu. Rev. Cond. Matter Phys. 6(1), 15 (2015). https://doi. org/10.1146/annurev-conmatphys-031214-014726

117. S. Korshunov, Sov. Phys. JETP 62(2), 301 (1985)

118. S.E. Korshunov, Phys. Uspekhi 49(3), 225 (2006). https://doi.org/10.1070/PU2006v049n03AB EH005838

119. K. Miyake, Prog. Theor. Phys. 69(6), 1794 (1983)

120. S. Kurihara, J. Phys. Soc. Jpn. 52(4), 1311 (1983). https://doi.org/10.1143/JPSJ.52.1311

121. A. Chubukov, A. Sokol, Phys. Rev. B 49, 678 (1994). https://doi.org/10.1103/PhysRevB.49.678

122. M.Y. Kagan, Phys. Uspekhi 37(1), 69 (1994). https://doi.org/10.1070/PU1994v037n01ABEH000 003

123. H. Ikegami, K. Kim, D. Sato, K. Kono, H. Choi, Y.P. Monarkha, Phys. Rev. Lett. 119(19), 195302 (2017). https://doi.org/10.1103/PhysRevLett.119.195302

124. L.V. Levitin, R.G. Bennett, A. Casey, B. Cowan, J. Saunders, D. Drung, T. Schurig, J.M. Parpia, Science 340(6134), 841 (2013). https://doi.org/10.1126/science.1233621

125. L.V. Levitin, R.G. Bennett, E.V. Surovtsev, J.M. Parpia, B. Cowan, A.J. Casey, J. Saunders, Phys. Rev. Lett. 111(23), 235304 (2013). https://doi.org/10.1103/PhysRevLett.111.235304

126. L.V. Levitin, R.G. Bennett, A. Casey, B. Cowan, J. Saunders, D. Drung, T. Schurig, J.M. Parpia, B. Ilic, N. Zhelev, J. Low Temp. Phys. 175(5-6), 667 (2014). https://doi.org/10.1007/s1090 9-014-1145-1

127. N. Zhelev, T.S. Abhilash, E.N. Smith, R.G. Bennett, X. Rojas, L. Levitin, J. Saunders, J.M. Parpia, Nat. Commun. 8, 15963 (2017). https://doi.org/10.1038/ncomms15963

128. L.V. Levitin, B. Yager, L. Sumner, B. Cowan, A.J. Casey, J. Saunders, N. Zhelev, R.G. Bennett, J.M. Parpia, Phys. Rev. Lett. 122(8), 085301 (2019). https://doi.org/10.1103/PhysRevLet t.122.085301

129. G.E. Volovik, Exotic properties of superfluid ${ }^{3}$ He (World Scientific, Singapore, 1992)

130. A. Casey, J. Parpia, R. Schanen, B. Cowan, J. Saunders, Phys. Rev. Lett. 92(25), 255301 (2004). https://doi.org/10.1103/PhysRevLett.92.255301

131. P. Sharma, A. Corcoles, R.G. Bennett, J.M. Parpia, B. Cowan, A. Casey, J. Saunders, Phys. Rev. Lett. 107(19), 196805 (2011). https://doi.org/10.1103/PhysRevLett.107.196805

132. Z. Tesanovic, M.V. Jaric, S. Maekawa, Phys. Rev. Lett. 57(21), 2760 (1986). https://doi. org/10.1103/PhysRevLett.57.2760

133. N. Trivedi, N.W. Ashcroft, Phys. Rev. B 38(17), 12298 (1988). https://doi.org/10.1103/PhysR evB.38.12298

134. A. Noury, J. Vergara-Cruz, P. Morfin, B. Placais, M.C. Gordillo, J. Boronat, S. Balibar, A. Bachtold, Phys. Rev. Lett. 122(16), 165301 (2019). https://doi.org/10.1103/PhysRevLett.122.165301

135. Z. Wang, J. Wei, P. Morse, J.G. Dash, O.E. Vilches, D.H. Cobden, Science 327(5965), 552 (2010). https://doi.org/10.1126/science. 1182507

136. H.C. Lee, O.E. Vilches, Z. Wang, E. Fredrickson, P. Morse, R. Roy, B. Dzyubenko, D.H. Cobden, J. Low Temp. Phys. 169(5-6), 338 (2012). https://doi.org/10.1007/s10909-012-0642-3 
137. B. Dzyubenko, H.C. Lee, O.E. Vilches, D.H. Cobden, Nat. Phys. 11(5), 398 (2015). https://doi. org/10.1038/nphys3302

138. L. Reatto, D.E. Galli, M. Nava, M.W. Cole, J. Phys, CondEN. Matter 25(44), 443001 (2013). https ://doi.org/10.1088/0953-8984/25/44/443001

Publisher's Note Springer Nature remains neutral with regard to jurisdictional claims in published maps and institutional affiliations. 